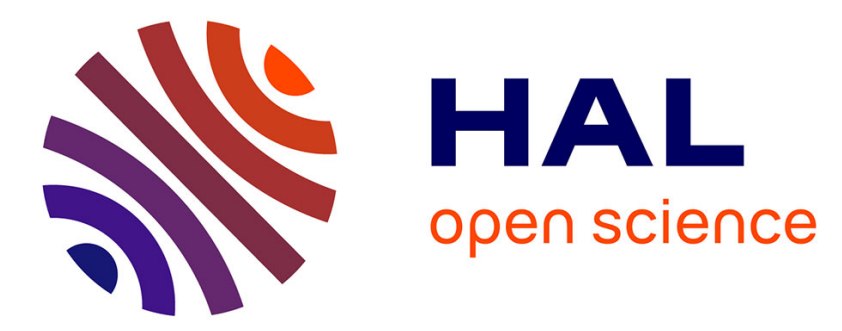

\title{
Increasing Attentional Load Boosts Saccadic adaptation
} Peggy Gerardin, Judith Nicolas, Alessandro Farne, Denis Pelisson

\section{To cite this version:}

Peggy Gerardin, Judith Nicolas, Alessandro Farne, Denis Pelisson. Increasing Attentional Load Boosts Saccadic adaptation. Investigative Ophthalmology \& Visual Science, 2015, 56, pp.6304-6312. 10.1167/iovs.14-16149 . hal-02196692

\section{HAL Id: hal-02196692 https://hal.science/hal-02196692}

Submitted on 29 Jul 2019

HAL is a multi-disciplinary open access archive for the deposit and dissemination of scientific research documents, whether they are published or not. The documents may come from teaching and research institutions in France or abroad, or from public or private research centers.
L'archive ouverte pluridisciplinaire $\mathbf{H A L}$, est destinée au dépôt et à la diffusion de documents scientifiques de niveau recherche, publiés ou non, émanant des établissements d'enseignement et de recherche français ou étrangers, des laboratoires publics ou privés. 


\title{
Increasing Attentional Load Boosts Saccadic Adaptation
}

\author{
Peggy Gerardin, ${ }^{1-3}$ Judith Nicolas, ${ }^{1,3}$ Alessandro Farnè,${ }^{1,3}$ and Denis Pélisson ${ }^{1,3}$ \\ ${ }^{1}$ ImpAct Team, Centre de Recherche en Neurosciences de Lyon, Bron, France \\ ${ }^{2}$ Stem-Cell and Brain Research Institute, Department of Integrative Neurosciences, Bron, France \\ ${ }^{3}$ UCBL Lyon University, Lyon, France
}

Correspondence: Peggy Gerardin, INSERM U846 Stem-Cell and Brain Research Institute, Department of Integrative Neurosciences, 18 , av. du Doyen Lépine 69500 Bron, France; peggy.gerardin@inserm.fr.

Submitted: November 26, 2014 Accepted: August 21, 2015

Citation: Gerardin P, Nicolas J, Farnè A, Pélisson D. Increasing attentional load boosts saccadic adaptation. Invest Ophthalmol Vis Sci.

2015;56:XXX-XXX. DOI:10.1167/ iovs.14-16149
Purpose. Visual exploration relies on saccadic eye movements and attention processes. Saccadic adaptation mechanisms, which calibrate the oculomotor commands to continuously maintain the accuracy of saccades, have been suggested to act at downstream (motor) and upstream (visuoattentional) levels of visuomotor transformation. Conversely, whether attention can directly affect saccadic adaptation remains unknown. To answer this question, we manipulated the level of attention engaged in a visual discrimination task performed during saccadic adaptation.

Methods. Participants performed low or high attention demanding orientation discrimination tasks on largely or faintly oriented Gabor patches, respectively, which served as targets for reactive saccades. Gabor patches systematically jumped backward during eye motion to elicit an adaptive shortening of saccades, and replaced $50 \mathrm{msec}$ later (100 $\mathrm{msec}$ in two subjects) by a mask. Subjects judged whether Gabors' orientation was "nearly horizontal" versus "nearly vertical" (low attention demanding) or "slightly left" versus "slightly right" (high attention demanding), or made no discrimination (control task).

Results. We found that the build-up and the retention of adaptation of reactive saccades were larger in the "high attention demanding" condition than in the "low attention demanding" and the no-discrimination control conditions.

ConcLusions. These results indicate that increasing the level of attention to the perceptual processing of otherwise identical targets boosts saccadic adaptation, and suggest that saccadic adaptation mechanisms and attentional load effects may functionally share common neural substrates.

Keywords: attentional load, target discrimination, reactive saccades, saccadic adaptation

\begin{abstract}
ttention and eye movements are two major components of $A_{\text {visual perception, both being necessary to select informa- }}$ tion from our rich visual environment. Although typically linked, spatial attention can move independently of eye movements, a capacity known as covert attention. ${ }^{1}$ The focus of covert attention can be measured as the spatially restricted areas where performance in visual detection or discrimination tasks is best. Attention typically can be driven by endogenous (e.g., verbal commands) or exogenous cues (e.g., spatially restricted stimuli) provided shortly before presentation of the target. ${ }^{1}$ Another form of exogenous shift of covert attention, called presaccadic shift of attention, takes place in absence of any cue, just before a saccade is elicited toward the vicinity of the target. ${ }^{2-5}$ According to the premotor theory of attention, ${ }^{6}$ covert shifts of visuospatial attention are nothing but an oculomotor activation (saccade preparation) without actual movement execution. A recent reappraisal of this theory suggests that it holds particularly well for the exogenous type of visual attention shift ${ }^{7}$ : indeed, presaccadic shifts of attention seem to be coupled with the saccade endpoint, rather than with the saccade target location ${ }^{8-11}$ (but see Ditterich et al. ${ }^{12}$ ), when those are dissociated by saccadic adaptation elicited by the target double-step paradigm. ${ }^{13}$ Note that, although the premotor theory predicts a bidirectional functional coupling between attention and action, evidence for a link from attention to oculomotor responses is scarce. The effects

exerted on saccade initiation by perceptual urgency, ${ }^{14}$ motivation, ${ }^{15}$ or concurrent distractor presentation ${ }^{11}$ may, in fact, occur at sensory level and, thus, only indirectly point at a functional coupling between attention and action. Another piece of evidence is the demonstrated effect on saccade curvature of visual distractors presented shortly before saccade execution, ${ }^{16-18}$ but the alternative explanation of low-level interaction between target and distractor visual signals cannot be dismissed. Recently, we have shown ${ }^{19,20}$ that adaptation of reactive saccades (RS, elicited by the sudden presentation of a visual target) by the target double-step paradigm engaged activity in the temporo-parietal junction (TPJ), whereas adaptation of voluntary saccades (VS, elicited while scanning a set of visual targets) activated the posterior intraparietal sulcus (pIPS). This specificity of adaptation-related network relative to saccade type is compatible with the proposed ventral and dorsal systems controlling, respectively, exogenous and endogenous shifts of attention. ${ }^{2,12,21}$ This last observation suggests a functional coupling between covert attention and saccadic adaptation.

To test this hypothesis, we studied whether experimentallycontrolled modulation of the subjects' attention can modify saccadic adaptation. We used this model of sensorimotor plasticity because it allows for automatic (without subject's awareness) changes of internal spatial parameters of the saccadic system in response to repeated visual target perturba- 
tion. $^{22}$ To vary the attentional demands, subjects had to perform either the saccadic adaptation alone, or to additionally discriminate the same visual stimuli used to elicit adaptation, but with two sets of instructions yielding two different levels of difficulty. The results showed that increasing the attentional demand enhanced saccadic adaptation.

\section{Materials And Methods}

\section{Subjects}

Eight subjects with normal or corrected-to-normal vision volunteered for this study ( 6 females; mean age \pm SD, $31 \pm$ 5). They performed the high-low attention conditions in a counter-balanced order (separated by at least 7 days). Eight additional subjects (one from the previous group, 6 females; mean age $\pm S D, 29.5 \pm 5$ ) participated in the no-discrimination task control experiment. All but one (author) were naive to the goal of the study. All procedures complied with the Ethical Principles of the Declaration of Helsinki.

\section{Experimental Set-Up and Stimuli}

Experiments were performed in a dark room. Subjects were sitting $57 \mathrm{~cm}$ away from a $140 \mathrm{~Hz}$ linearized computer screen $\left(30^{\circ} \times 40^{\circ}\right)$, with their head stabilized by a chin rest, cheekbone rests and frontal support. An infrared tracker (Eyelink 1000, SR Research, Ottawa, Ontario, Canada) with a frequency of $500 \mathrm{~Hz}$ and a spatial resolution of $0.05^{\circ}$ was used to record binocular eye movements. Laboratory-made software allowed on-line monitoring of calibrated eye movements, saccade-contingent triggering of the visual stimulation, and storage of eyemovement data for off-line analysis.

Stimuli were created with Matlab R2010b (available in the public domain at http://www.mathworks.com/) and displayed with the Visual Stimuli Generation system (CRS, Cambridge, United Kingdom) on a gray background $\left(22 \mathrm{~cd} / \mathrm{m}^{2}\right)$. Stimuli consisted of two target categories (Fig. 1b): a plaid (superimposition of two orthogonal Gabor patches) served as initial target in all blocks and, in adaptation phase, a 2-cycle/deg Gabor tilted at different angles $\left( \pm 2.5^{\circ}\right.$ with respect to the vertical, $\pm 2.5^{\circ}$ with respect to the horizontal) served as secondary target. All targets had a diameter of $2^{\circ}$. A black dot $\left(0.6^{\circ}\right.$ diameter $)$ served as fixation point.

\section{Procedure}

In the main experiment, the two discriminative conditions ("Low" [LAD] and "High" [HAD] Attention Demanding) differed from each other only in the task difficulty related to different instructions, the visual stimuli and oculomotor tasks being identical. They comprised three saccadic adaptation blocks, and pre- and postadaptation blocks with no target jump (description in Fig. 1a caption).

Pre- and postadaptation blocks, specifically suited to assess adaptive changes of motor commands (see the study of Bastian $^{23}$ ), consisted each of 24 trials (12 per direction, in a randomized order) of reactive (RS) and voluntary (VS) saccades. In these blocks, RS trials started with a central fixation point that extinguished after a variable delay (500-1100 ms) while a plaid target that subjects were required to saccade appeared at $\pm 16^{\circ}$. Upon saccade detection (velocity threshold, $80^{\circ}-90^{\circ} / \mathrm{s}$ ), the target was made to disappear leaving a blank screen. Voluntary saccade trials also started with the fixation point, but located $4^{\circ}$ above the screen center. After $1500 \mathrm{~ms}$, two plaid targets appeared, one located at the screen center and the other located laterally at $\pm 16^{\circ}$. After $500 \mathrm{~ms}$, a circle surrounding the fixation point appeared signaling subjects first to make a vertical saccade toward the central target and then to voluntarily initiate a horizontal saccade toward the lateral target. All targets and fixation point disappeared upon detection of the horizontal VS (velocity threshold, $80^{\circ}-90^{\circ} / \mathrm{s}$ ). Note that no visual feedback (no Gabor) was provided in these blocks, as the plaid disappeared upon saccade initiation.

The adaptation phase consisted of 3 blocks of 48 RS trials (12 rightward and 36 leftward saccades in a randomized order). During each trial, a central fixation point appeared for a random duration (500-1100 ms) and then turned off while simultaneously a plaid target appeared at $\pm 16^{\circ}$. Subjects were required to saccade the target and as soon as the RS was detected (velocity threshold, $80^{\circ}-90^{\circ} / \mathrm{s}$ ) this target disappeared and was replaced by one of the four possible Gabors (Fig. 1b). For six subjects, the Gabor target was displayed for $50 \mathrm{~ms}$ and was replaced by a white noise dots mask ( $50 \mathrm{~ms}$ duration) covering the whole display to shorten the target visual persistence. For two subjects, due to weaker discrimination abilities measured in pilot testing, the duration of the Gabor target and of the mask were increased to $100 \mathrm{msec}$. As the screen returned blank, subjects were asked to respond whether the appearing Gabor was nearly vertical or nearly horizontal (LAD), or was tilted slightly left or slightly right (HAD). In all rightward trials, the Gabor target was presented at the same location as the plaid target, whereas in the leftward trials, the Gabor was systematically shifted toward the center to elicit an amplitude shortening adaptation. The blank interval was dependent on saccade latency: as subjects were instructed to move their eyes back to the center as soon as the blank interval started, this insured that no rightward saccade toward a visible fixation point was made. The intrasaccadic target step represented $25 \%$, $32.5 \%$, and $40 \%$ of initial target eccentricity for the first, second, and third block of trials, respectively. The Gabor 50 ms duration was chosen as previously proven sufficient to induce saccade adaptation, ${ }^{24}$ whereas longer duration would have reduced the discrimination task difficulty, as determined by pilot tests. All participants shortly practiced the discrimination task ( 15 trials) without target jump (similar to trials with target in the right hemifield) and had short breaks (1-2 $\mathrm{mn}$ ) between adaptation blocks during the main experiment.

In the no-discrimination control experiment, subjects performed a single session with identical stimuli as in the main experiment, but did not perform any discrimination task on the Gabors (see Supplementary Material).

\section{Eye Data Analysis}

The "cyclopean" eye position was first derived by averaging the two eye position waveforms. Horizontal saccade amplitude was computed as the difference of horizontal eye position between saccade onset and termination. Saccade gain was computed as the ratio between saccade amplitude and target eccentricity (distance relative to initial eye position). Gain change was calculated as the difference between each individual saccade gain in the adaptation and post phases, and the mean gain in the corresponding prephase. For the adaptation phase, accuracy of the primary saccade was measured as the distance between its endpoint and the center of the backward stepped target, resulting in saccadic error in degree. Finally, the speed of adaptation was assessed for each subject and each adaptation block, by measuring the slope of the linear relationship between saccadic gain and trial number 
a

\section{Pre Phase}

\begin{tabular}{|c|c|}
\hline \multicolumn{1}{|c|}{ VS } & \multicolumn{1}{c|}{ RS } \\
24 trials & 24 trials \\
12 leftward & 12 leftward \\
12 rightward & 12 rightward \\
\hline
\end{tabular}

No intra-saccadic step

VS: Voluntary Saccades

RS: Reactive Saccades
Adaptation Phase

\begin{tabular}{|c|c|c|}
\hline intra-saccadic step & intra-saccadic step & intra-saccadic step \\
of $25 \%$ & of $32.5 \%$ & of $\mathbf{4 0 \%}$ \\
48 trials & 48 trials & 48 trials \\
36 leftward (Adapted) & 36 leftward (Adapted) & 36 leftward (Adapted) \\
12 rightward (Control) & 12 rightward (Control) & 12 rightward (Control) \\
\hline
\end{tabular}

RS adaptation (intra-saccadic step)

+ Discrimination task
Post Phase

\begin{tabular}{|c|c|}
\hline \multicolumn{1}{|c|}{ RS } & \multicolumn{1}{c|}{ VS } \\
24 trials & \multicolumn{1}{c|}{24 trials } \\
12 leftward & 12 leftward \\
12 rightward & 12 rightward \\
\hline
\end{tabular}

No intra-saccadic step

VS: Voluntary Saccades

RS: Reactive Saccades

Initial Target
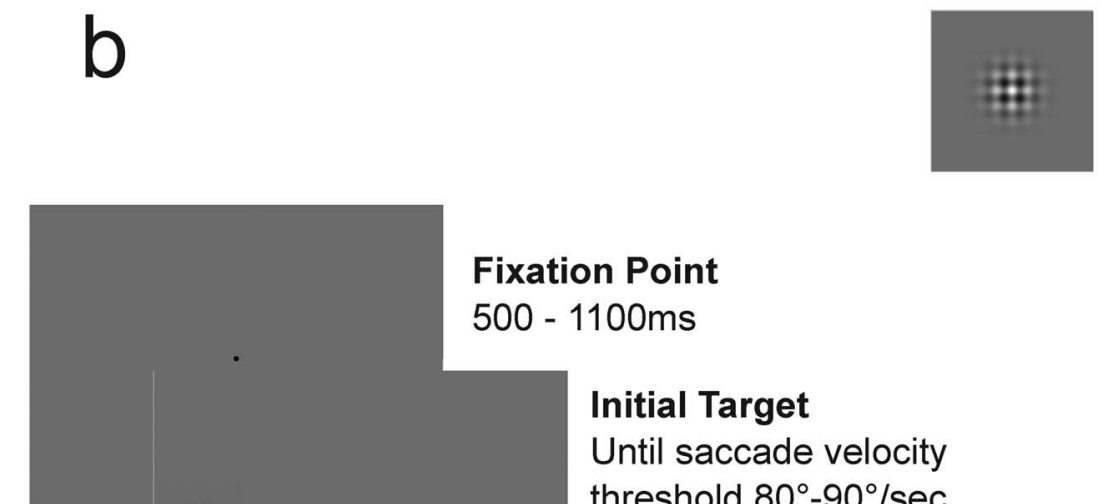

\section{Fixation Point}

$500-1100 \mathrm{~ms}$
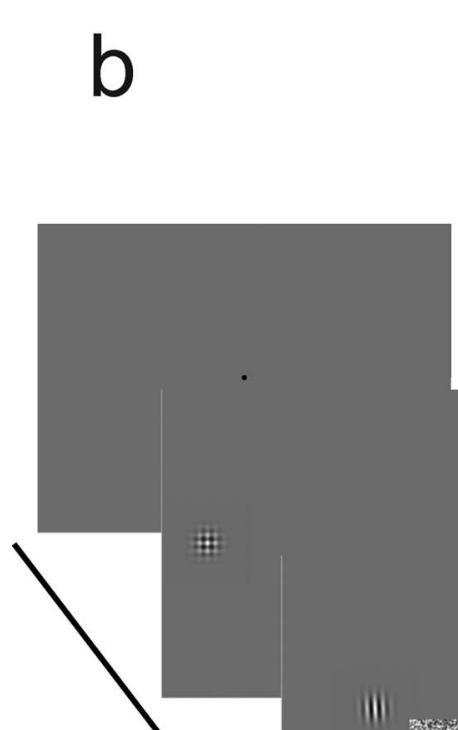

Initial Target

Until saccade velocity

threshold $80^{\circ}-90^{\circ} / \mathrm{sec}$

Secondary Target

$50 \mathrm{~ms}^{*}$

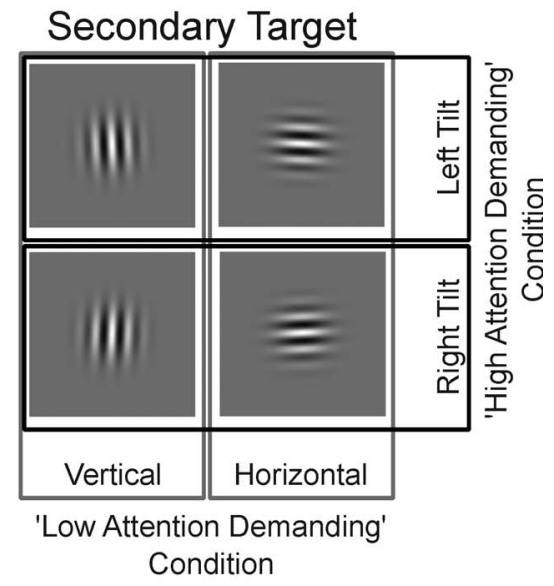

Surrounded FP

Subject's

response (Key)

Mask

$50 \mathrm{~ms}^{*}$

Time

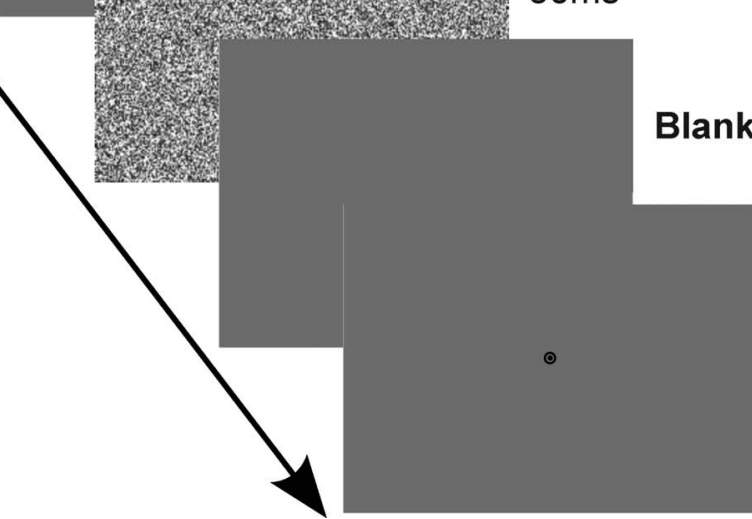

FiguRE 1. Experimental procedure, temporal sequence and stimuli. (a) Schematic description of the experimental procedure. Adaptation phase consisted of 48 RS trials (12 rightward saccades and 36 leftward saccades in a randomized order). Pre- and postadaptation phases each consisted of 24 trials (12 rightward saccades and 12 leftward saccades in a randomized order) of RS and VS saccades. (b) Left: Adaptation trial temporal sequence. Subjects were asked first to fixate the center and then to make a saccade toward the initial target (plaid target consisting of two superimposed Gabor patches, right). As soon as the saccade was detected (velocity threshold $80^{\circ}-90^{\circ} / \mathrm{s}$ ), the secondary target appeared for $50 \mathrm{~ms}\left({ }^{*} 100 \mathrm{msec}\right.$ in two subjects, see Methods). This target was randomly selected among the four Gabor patches shown on the right (2-cycle/deg, tilted $+2.5^{\circ}$ or $-2.5^{\circ}$ with respect to the vertical or to the horizontal). For leftward saccades, the secondary target was stepped relative to the initial target by $25 \%, 32.5 \%$, or $40 \%$ in adaptation blocks 1,2 , and 3 , respectively. For rightward saccades, the secondary target appeared at the same location as the initial target $\left(16^{\circ}\right)$. A mask then appeared for 50 ms $\left({ }^{*} 100\right.$ msec in two subjects, see Methods) immediately at target offset. At the end of each trial, when the surrounded fixation point appeared, subjects were asked to discriminate the tilt of the Gabor according to two different instructions: vertical or horizontal tilt in LAD versus left or right tilt in HAD. Note that in preand postadaptation phases of RS (not shown), the sequence of events was the same until the saccade was detected at which point the screen was blanked until the start of the next trial (no secondary target and no discrimination: see main text for more details about the procedure and for the VS pre- and postadaptation trials). 
(gain change slope). Approximately 2\% of trials were excluded from analyses because of blinks or noisy eye position signal. All statistical analyses (1-way ANOVAs with adaptation blocks as factor [3 levels] performed separately for each session, 2-way ANOVAs [3 adaptation blocks $\times 2$ sessions], and post hoc paired $t$-tests) were performed with Matlab R2010b Statistical Toolbox (available in the public domain at http://www. mathworks.com/).

\section{Results}

First, we computed for each subject the percentage of correct responses in each condition. The mean percentage of correct responses was significantly lower in HAD $(75.7 \pm 8 \%)$ than in LAD (91.3 $\pm 6 \%$; 2-way ANOVA, significant effect of condition [HAD, LAD] $F[1,42]=15.6 ; P<0.001)$. This difference is consistent with a higher level of difficulty of the perceptual task in HAD than in LAD, and, indeed, all subjects reported at debrief that they needed to concentrate more in the former task.

Second, we compared saccadic data between LAD and HAD for pre-/postadaptation phases and for adaptation phases.

\section{Pre-/Postadaptation Phases}

Figure 2 represents the mean gain change (post minus pre) of RS (Fig. 2a) and VS (Fig. 2b). As seen on the Figure, there was a reduction of gain for leftward saccades but no change for rightward saccades. This unidirectional effect is compatible with the well-known direction-specificity of saccadic adaptation (see review of Pelisson et al. ${ }^{22}$ ). Crucially, these adaptation-related reductions of leftward saccades gain were larger in HAD than in LAD. Indeed, there was a significant difference of gain change between the two conditions, for RS (paired $t$-test, $t[7]=3.02, P<0.05)$ and for VS $(t[7]=2.5, P<$ 0.05 ; see also individual gain and gain changes of RS and VS in Supplementary Tables S3 and S4). However, the transfer of RS adaptation to VS, as calculated by (VS gain change/RS gain change) $\times 100$, was not significantly higher in HAD $(60 \%)$ than in $\operatorname{LAD}(35.5 \% ; t[7]=-1.86, P=0.1$; see also the intra-subject variability of post-VS gain in sessions in Supplementary Table S2). Importantly, the discrimination task did not by itself modify saccade gain, as disclosed by the lack of significant gain difference for rightward saccades between pre/post and RS adaptation phases (1-way ANOVA, $F[7,4]=0.54, P=0.71$ ).

Thus, pre- versus postadaptation gain changes reveal that the retention of RS adaptation significantly increased with the level of attention load. These values also exceeded those observed in the no-discrimination experiment where adaptation was elicited in a new group of subjects (with the exception of 1 participant) in the absence of any discrimination task (gain change, $-0.034[ \pm 0.05]$, see Supplementary Data and Supplementary Table S1 for statistical results and mean gain values, respectively).

\section{Saccadic Adaptation Phases}

To test whether the level of attention load also modified the time-course of adaptation, we plotted in Figure $3 a$ the RS gain data during the three adaptation blocks. The individual mean gain plot (Fig. 3b) illustrates that, in both conditions, the gain decreased progressively as a function of trial number from a common baseline value (no difference of preadaptation RS gain between HAD and $\operatorname{LAD}(t[7]=0.98, P=0.35)$, in accordance with the backward intrasaccadic target step. Moreover, this gain decrease appeared to differ between conditions, as more clearly illustrated by the mean gain change values computed in each adaptation block (Fig. 3a): the gain change was systematically higher in HAD than in LAD.
Indeed, a 2-way ANOVA showed a significant effect of condition on saccadic gain change $(F[1,42]=13.1, P<0.01)$, and post hoc paired $t$-test performed for each adaptation block showed a significant difference between conditions in the $25 \%$ block $(t[7]=2.7, P<0.05)$, in the $32.5 \%$ block $(t[7]=2.8, P<$ $0.05)$, and in the $40 \%$ block $(t[7]=4.8, P<0.01)$. In contrast, the gain change of rightward saccades (i.e., performed in the nonadapted direction) did not show any difference between conditions $(-0.0368 \pm 0.01$ for $\mathrm{LAD}$ versus $-0.0121 \pm 0.01$ for $\mathrm{HAD}, F[1,42]=2.89, P=0.09$; see also individual mean gain plot in the non-adapted right hemifield [Supplementary Fig. S1]).

We then investigated the speed of adaptation by computing for each subject and each adaptation block the gain change slope (see Methods). As shown in Figure 4, the negative value of gain change slope (reflecting the adaptation-related decrease of saccade gain) was nearly constant for all three blocks of LAD. This is confirmed by the absence of significant "Block" effect of the 1-way ANOVA $(F[2,21])=0.064, P=0.94)$. In contrast, in HAD, there was a significant "Block" effect $(F[2,21]=10.04, P<0.001)$, revealing a progressive increase from blocks $25 \%$ to $40 \%$ (i.e., a reduction of the negative slope, Fig. 4a, right). We next asked whether attention load also has an effect on the accuracy of adapted primary saccades (see Fig. 4c). A 2-way ANOVA testing leftward saccadic accuracy revealed a significant effect of condition $(F[1,42]=11.6, P<$ $0.01)$. Post hoc paired $t$-test revealed that conditions differed for the $25 \%$ block $\left(2.48^{\circ}[ \pm 0.69]\right.$ in LAD versus $1.57^{\circ}[ \pm 0.77]$ in HAD $t[7]=2.7, P<0.05)$, for the $32 \%$ block $\left(2.87^{\circ}[ \pm 0.95]\right.$ vs. $\left.1.83^{\circ}[ \pm 0.7], t[7]=2.7, P<0.05\right)$ and for the $40 \%$ block $\left(2.86^{\circ}[ \pm 1.05]\right.$ vs. $\left.2.2^{\circ}[ \pm 1.05], t[7]=4.8, P<0.05\right)$.

As a first step toward comparing the perceptual and oculomotor performance of our subjects, we plotted in Figure $4 \mathrm{~b}$ the percentage of correct responses in the discrimination task. We found a similar pattern of change between Blocks as for the gain change slope: the 1-way ANOVA revealed no significant effect of the "Block" factor for $\operatorname{LAD}(F[2,21]=1.36$, $P=0.27)$, but disclosed a significant effect for $\operatorname{HAD}(F[2,21]=$ $6.56, P<0.01$ ). For $\mathrm{HAD}$, the mean perceptual performance increased from block $25 \%$ to block $40 \%$, whereas the saccade gain decreased less and less rapidly. Note further that for rightward saccade trials (control), subjects did not show any significant change across blocks of their perceptual performance for either condition (LAD, $F[2,21]=0.4, P=0.73$; HAD, $F[2,21]=0.06, P=0.93$ ) mean performance levels shown as dotted lines in Figure $4 \mathrm{~b}$. The pattern of changes over blocks in gain slopes and discrimination performance for HAD (Figs. 4a, 4b) could support the possibility of a trade-off whereby subjects adapt best when their discrimination performance is least. Lower perceptual performance being unlikely to cause such an increased rate of adaptation, we suggest it rather reflects an increased deployment of attentional resources due to perceptual task difficulty, which boosts saccadic error correction mechanisms. Since a significant change of aftereffects is detected between conditions, we believe that these mechanisms involve sensorimotor saccadic plasticity.

We then tested for potential correlations between perceptual and oculomotor performances. We first correlated the percentage of correct responses determined for each subject and each block to the respective gain change slopes. Whereas no correlation was found for LAD $(\rho=-0.09, P=0.54)$, a significant correlation was found for $\operatorname{HAD}(\rho=0.35, P<0.05)$. This observation is consistent with the positive relationship between mean gain slope and correct responses (increase of correct responses as a function of less negative gain slopes) observed in Figures 4a, 4b (right panels). We then correlated subjects' perceptual performance and saccadic error and found 
a

Pre/Post Phase for Reactive Saccades (RS)
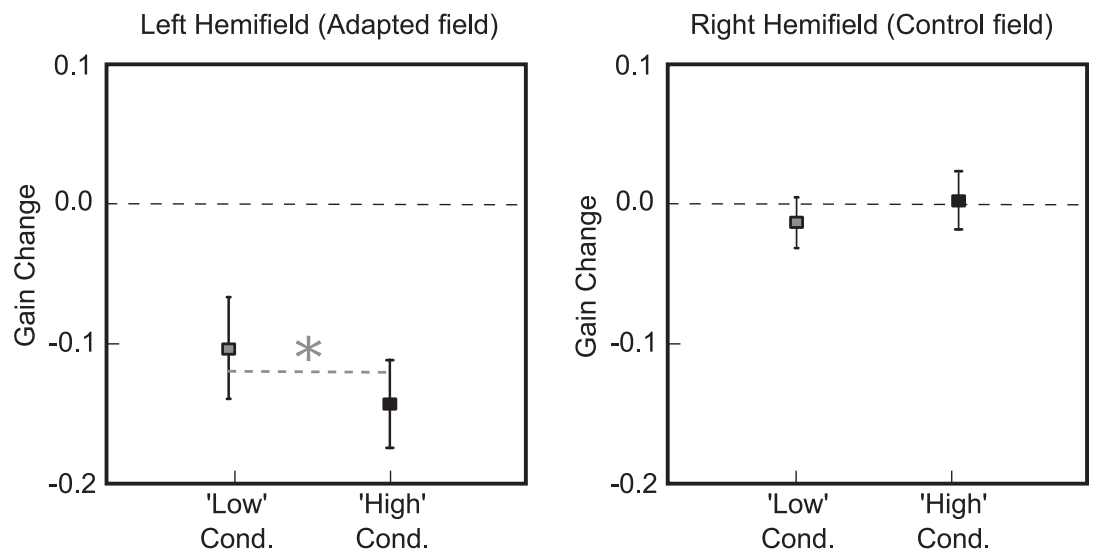

b

Pre/Post Phase for Voluntary Saccades (VS)

Left Hemifield (Adapted field)

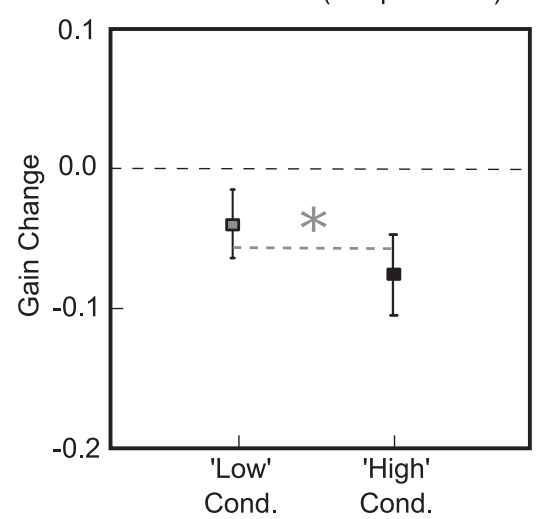

Right Hemifield (Control field)

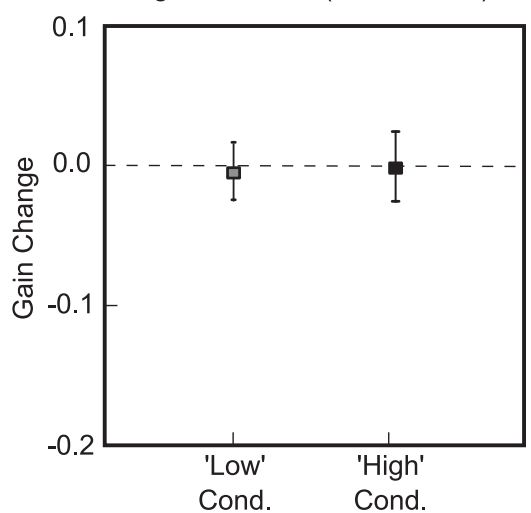

Figure 2. Mean Gain Change between Pre and Post phases for Reactive (RS) and Voluntary (VS) saccades. (a) RS mean ( \pm SD) saccadic gain change (Postphase - Prephase), for LAD (gray squares) and HAD (black squares) conditions. Left (adapted) hemifield: mean gain change $=-0.10$ ( \pm 0.034 ) for LAD, -0.13 ( \pm 0.033$)$ for HAD. Star symbol: significant difference between conditions. No gain change has been noticed in the right (control) hemifield. (b) VS mean ( $\pm \mathrm{SD}$ ) saccadic gain change (Postphase - Prephase), for LAD (gray squares) and HAD (black squares). Left (adapted) hemifield: mean gain change $=-0.04( \pm 0.028)$ for LAD (significant difference of gain between Pre- and Postphases, $t[7]=3.6, P<0.05$ ), -0.08 $( \pm 0.034)$ for HAD (significant difference of gain between Pre- and Postphases, $t[7]=6.4, P<0.001$ ). Star symbol: significant difference between conditions. No gain change has been noticed in the right (control) hemifield.

no significant correlation for saccadic error (LAD, $\rho=-0.04, P$ $=0.76$; $\mathrm{HAD}, \rho=0.08, P=0.43)$.

Finally, we checked whether other oculomotor parameters varied as a function of the attentional load levels. A 2-way ANOVA showed no significant difference in latency between LAD $(206 \pm 16 \mathrm{~ms})$ and HAD $(202 \pm 18 \mathrm{~ms}, F[1,42]=1.14, P$ $=0.18)$, nor in peak velocity $(F[1,42]=1.07, P=0.3)$. A 1 -way ANOVA did not show any significant difference in saccade latency between "Pre/Post"-RS phases and the 3 RS adaptation blocks (LAD, $F[4]=0.39, P=0.81 ; \mathrm{HAD}, F[4]=0.67, P=0.61$, Fig. 3c).

\section{Discussion}

By varying the level of attention necessary to discriminate target stimuli used to elicit adaptation of RS, we found that the amount and speed of RS adaptation were larger in the HAD task than in the LAD task.

\section{Effect of Attentional Load on Saccadic Adaptation}

The comparison of perceptual performance between the two conditions confirms that HAD was more difficult than LAD. In particular, in $\mathrm{LAD}$, the amount of correct responses was stable 
a

Saccadic Adaptation Phase: Mean Gain Change for Adapted (Left) Hemifield
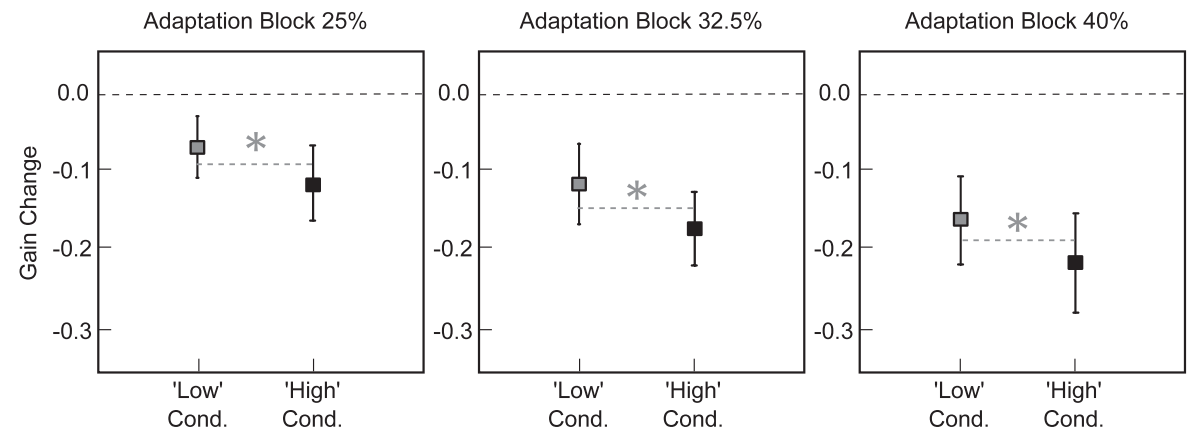

b

Pre/Post and Adaptation Phases: Saccades in the Adapted (Left) Hemifield.

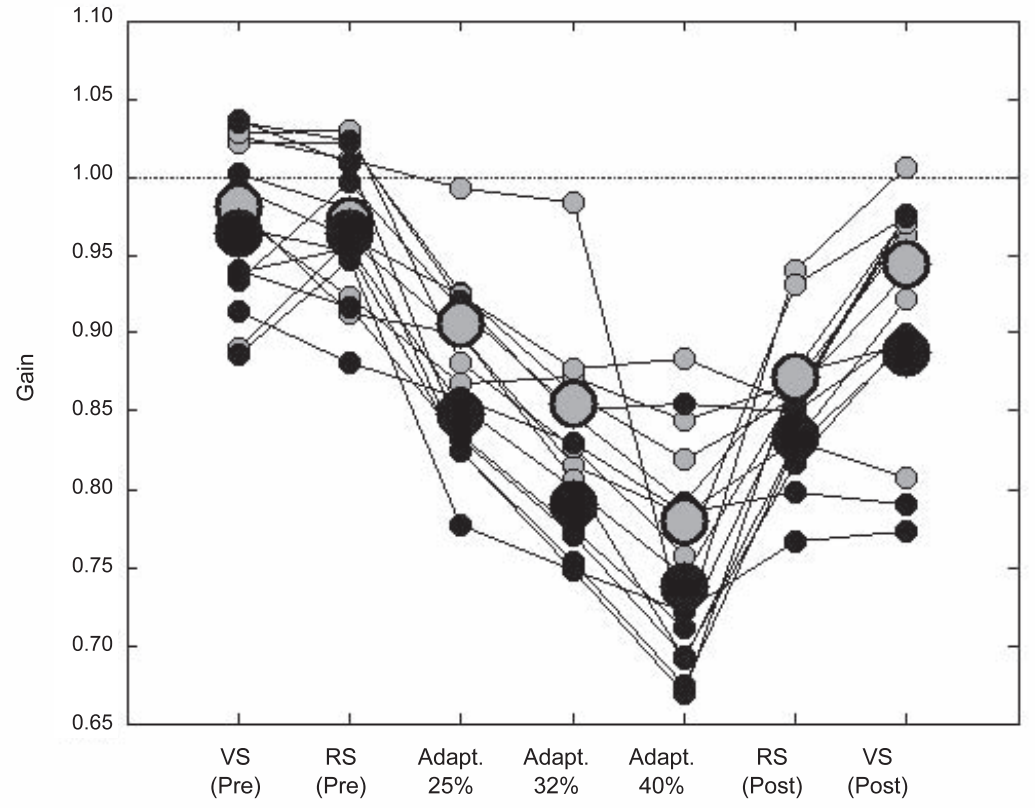

O 'Low Attention Demanding' Condition

- 'High Attention Demanding' Condition

C Pre/Post and Adaptation Phases: S1 Latencies in the Adapted (Left) Hemifield.

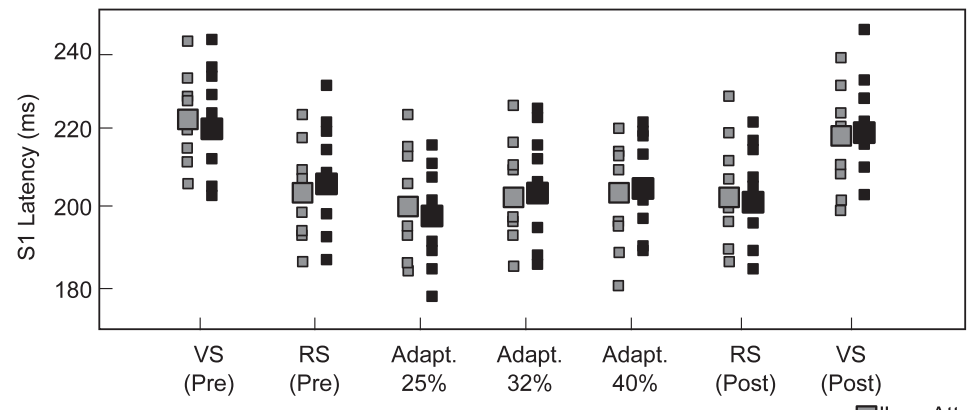

'Low Attention Demanding' Condition

'High Attention Demanding' Condition

Figure 3. Adaptation phase. (a) Mean ( \pm SD) saccadic gain change ([saccade amplitude/initial target eccentricity] - Prephase) in Adaptation phase (intrasaccadic step of 25\% [left panel], 32.5\% [middle panel], 40\% [right panel] of the initial target eccentricity), for LAD (gray squares) and HAD (black squares) conditions. Left (adapted) hemifield: mean gain for $\mathrm{LAD}=0.9$ ( $\pm 0.043,25 \%$ block), 0.85 ( $\pm 0.06,32.5 \%$ block), 0.77 ( $\pm 0.07,40 \%$ block); mean gain for $\mathrm{HAD}=0.84( \pm 0.05,25 \%$ block $), 0.79( \pm 0.05,32.5 \%$ block $), 0.74( \pm 0.07,40 \%$ block). Star symbol: significant difference between conditions. Right (control) hemifield: mean gain for $\mathrm{LAD}=0.94( \pm 0.02)$ for all blocks; Mean gain for $\mathrm{HAD}=0.94( \pm 0.03)$ for all blocks. (b) Saccadic gain (saccade amplitude/ initial target eccentricity) plotted separately in LAD and HAD sessions as a function of trials blocks: Pre-VS, Pre-RS, Adaptation 25\%, Adaptation 32.5\%, Adaptation 40\%, Post-RS and Post-VS. Each small circle represents the mean saccadic gain in each subject; large circles represent grand means across all subjects. LAD: gray circles; HAD: black circles. (c) Saccadic latencies (ms) plotted separately in LAD and HAD sessions as a function of trials blocks: Pre-VS $(\mathrm{LAD}=222[ \pm 11.6], \mathrm{HAD}=220$ [ \pm 12$])$, Pre-RS $(\mathrm{LAD}=204$ [ \pm 12.8$], \mathrm{HAD}=206$ [ \pm 12.9$])$, Adaptation $25 \%(\mathrm{LAD}=202[ \pm 14.6], \mathrm{HAD}=199$ [ \pm 14.3$])$, Adaptation 32.5\% $(\mathrm{LAD}=201[ \pm 14], \mathrm{HAD}=204[ \pm 14.1])$, Adaptation $40 \%(\mathrm{LAD}=204[ \pm 12.9], \mathrm{HAD}=205[ \pm 11.8])$, Post-RS $(\mathrm{LAD}=204[ \pm 14], \mathrm{HAD}=$ $202[ \pm 13.2])$ and Post-VS $(\mathrm{LAD}=221[ \pm 12.6], \mathrm{HAD}=222[ \pm 11.5])$. Each square represents mean across all subjects. LAD, gray squares; HAD, black squares. 
Gain Slopes, \% Correct Responses and Saccadic Errors as a function of Blocks
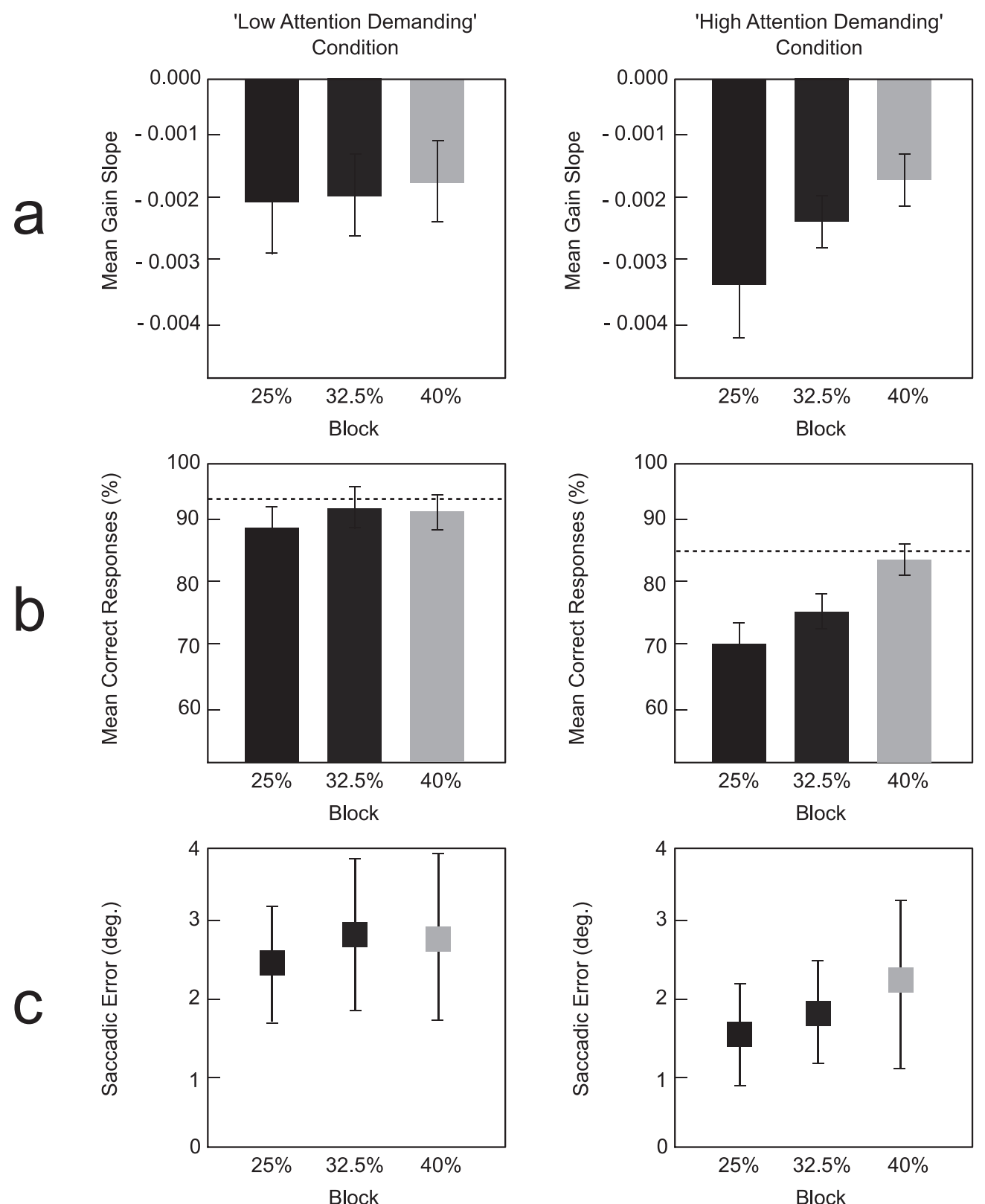

FiguRE 4. Mean gain slopes, mean correct responses (\%) and saccadic errors during adaptation phase. (a) Mean gain slopes (= slope of the relationships between saccadic gain and trial number for each subject in each block) as a function of blocks: $-0.0021( \pm 0.00081)$ for $25 \%,-0.002$ $( \pm 0.0007)$ for $32.5 \%$, and 0.0018 ( \pm 0.0008$)$ for $40 \%$ in the LAD condition (left). Mean gain slopes as a function of blocks: -0.0034 ( \pm 0.0006 ) for $25 \%,-0.0023( \pm 0.0003)$ for $32.5 \%$, and $0.0018( \pm 0.0004)$ for $40 \%$ in the HAD condition (right). (b) Mean correct responses (\%) as a function of blocks: $88.50( \pm 3.50)$ for $25 \%, 93.0( \pm 2.80)$ for $32.5 \%$, and 92.50 ( \pm 2.50$)$ for $40 \%$ for LAD (left). Mean correct responses (\%) as a function of blocks: 69.60 ( \pm 2.60 ) for $25 \%, 75.10$ ( \pm 4.30 ) for $32.5 \%$, and 82.20 ( \pm 3.40$)$ for $40 \%$ for HAD (right). Black dashed line show corresponding mean correct responses (\%) for all blocks in the right hemifield. A 2-way ANOVA performed on the correct responses in the right hemifield showed no effect of condition (HAD or LAD, $F[1,2]=1.43, P=0.23)$ and no effect of adaptation phase $(25 \%, 32 \%$, and $40 \%, F[1,2]=1.13, P=0.33)$, nor any interaction $(F[1,2]=1.18, P=0.31)$ between factors, suggesting that the variability mainly emerges at the interindividual level. (c) Saccadic errors (degree) as a function of blocks. The $y$-axis 0 value indicates the final target position. Filled squares: LAD, 2.48 ( \pm 0.69$)$ for $25 \%, 2.87( \pm 0.95)$ for $32.5 \%$, and 2.86 $( \pm 1.05)$ for $40 \%$. $\mathrm{HAD}, 1.57( \pm 0.77)$ for $25 \%, 1.83( \pm 0.7)$ for $32.5 \%$, and $2.2( \pm 1.05)$ for $40 \%)$.

over time and similar for the left (shifting) and the right (stationary) targets. In HAD, instead, the discrimination performance for left targets was lower, particularly at the beginning of the adaptation phase, and increased over time to reach the same level as for right targets. This increase in performance during HAD could, in principle, be related indirectly to the observed faster adaptation, yielding a lower error of primary saccades (i.e., a smaller retinal eccentricity of the Gabor). In fact, saccadic error did differ between the two conditions, being significantly smaller in HAD compared to 
LAD. This difference in saccade accuracy was present already in the first adaptation block, in which the difference of discrimination performance between conditions was highest. In addition, the time-course of saccade accuracy and discrimination performance over the 3 adaptation blocks are not parallel. Finally, there was no within-block correlation between perceptual performance and saccadic error in either condition. These observations suggested that the increase of performance during HAD is not causally related to changes in primary saccade accuracy. Thus, the significant correlation across trial blocks between this increase of performance and the adaptation slopes, with higher attentional load (indexed by lower perceptual performance) yielding higher adaptation rate, supports a causal relationship from attention load to adaptation efficacy.

In conclusion, higher attentional load required by visual processing of the saccadic target could boost either the processing of target error signals, necessary to elicit adaptation, and/or the plasticity processes underlying saccade adaptive modifications. We discuss below recent data supporting the existence of a coupling between attention and adaptation, and then the possible neural substrates of this coupling.

\section{Coupling Between Saccadic Adaptation and Attention/Visual Perception}

By showing a direct effect of attention on adaptation, our findings support and extend the notion of a functional coupling between covert attention and saccadic adaptation. Ditterich et al. ${ }^{21}$ previously compared the efficacy of saccadic adaptation in different visual background conditions. They found that intrasaccadic shifts of the background do not influence adaptation of saccades toward a small target, but do influence saccadic adaptation when a $4.8^{\circ}$ annulus is used as a target, and concluded that in the latter case the error signal inducing adaptation increased because the attentional focus was commensurate with the target size. In a recent study, Khan et $\mathrm{al}^{25}$ investigated the effect on saccade amplitude of flashing a distractor near a peripheral target, just after the completion of the saccade to this target. When flashed repeatedly at a less eccentric location than the target, and even though not eliciting any saccade, the distractor generated a progressive reduction of saccade amplitude, akin to saccadic adaptation. The authors concluded that the covert shift of exogenous attention elicited by the distractor could act as an error signal for saccade adaptation. This conclusion is consistent with another recent finding reported by Schütz et al. ${ }^{26}$ that saccade adaptation can be induced in a natural perceptual task without any bottom-up error signals (no target step). Indeed, adaptive changes of saccades performed toward a protracted target during a perceptual task were elicited by systematically placing a visual item to be discriminated (letter) at an eccentric location relative to the target center-of-gravity where the nonadapted saccade naturally lands. Thus, saccadic adaptation could be elicited by top-down information about the expected location of interest within a stationary visual target; therefore, possibly resulting from endogenous attention mechanisms. Finally, the likely best evidence so far for a link between attention and saccadic adaptation has been provided by McFadden et al. ${ }^{27}$ : these authors showed that it is possible to adapt covert attention shifts elicited in a Posner-like cued discrimination task and that such adaptation of attention transfers to saccades.

\section{Possible Neural Substrates}

The overlap of oculomotor and attention neural systems has provided a strong support to the premotor theory of spatial attention. Neuroimaging and transcranial magnetic stimulation (TMS) studies in humans have shown that attending covertly to a peripheral location or preparing to move the eyes toward this location activates the same neural network of frontoparietal regions ${ }^{7,28}$ which in turn modulate the activity of visual areas, such as MT and V4 (see prior review ${ }^{29}$ ). However, scarce evidence exists on the effects of attentional load on these spatial attention and saccade neural systems. A notable exception is an fMRI study ${ }^{30}$ which showed that attentional load, controlled by varying the number of simultaneously attended moving targets, activates several parietal and frontal cortical areas, including those involved in saccadic adaptation (Pre-Central Sulcus [PreCS] and intraparietal sulcus [IPS]), as reported in our previous fMRI and TMS studies. ${ }^{19,20}$ Therefore, one may speculate that the high attentional load in our study led to an increased activity of PreCS and IPS.

To conclude, the present study demonstrated that merely increasing the attentional load to the saccade target can boost saccadic adaptation. This coupling suggests that saccadic adaptation and visual attention mechanisms may share common neural substrates.

\section{Acknowledgments}

The authors thank Christian Urquizar and Romeo Salemme for their help with the eye-movement and stimuli software.

Supported by FRM, and performed within the framework of the LABEX CORTEX (ANR-11-LABX-0042) of Université de Lyon, within the program "Investissements d'Avenir" (ANR-11-IDEX0007), operated by the French National Research Agency (ANR).

Disclosure: P. Gerardin, None; J. Nicolas, None; A. Farnè, None; D. Pélisson, None

\section{References}

1. Posner MI. Orienting of attention. Quart J Exp Physiol. 1980; 32:3-25.

2. Deubel H, Schneider WX. Saccade target selection and object recognition: evidence for a common attentional mechanism. Vision Res. 1996;36:1827-1837.

3. Kowler E, Anderson E, Dosher B, Blaser E. The role of attention in the programming of saccades. Vision Res. 1995; 35:1897-1916.

4. Hoffman JE, Subramaniam B. The role of visual attention in saccadic eye movements. Percept Psychophys. 1995;57:787795.

5. Jonikaitis D, Theeuwes J. Dissociating oculomotor contributions to spatial and feature-based selection. J Neurophysiol. 2013;110:1525-1534.

6. Rizzolatti G, Riggio L, Dascola I, Umilta C. Reorienting attention across the horizontal and vertical meridians: evidence in favor of a premotor theory of attention. Neuropsychologia. 1987;25:31-40.

7. Smith DT, Schenk T. The Premotor theory of attention: time to move on? Neuropsychologia. 2012;50:1104-1114.

8. Collins $\mathrm{T}$, Dore-Mazars $\mathrm{K}$. Eye movement signals influence perception: evidence from the adaptation of reactive and volitional saccades. Vision Res. 2006;46:3659-3673.

9. Dore-Mazars K, Collins T. Saccadic adaptation shifts the presaccadic attention focus. Exp Brain Res. 2005;162:537-542.

10. Collins T, Heed T, Dore-Mazars K, Roder B. Presaccadic attention interferes with feature detection. Exp Brain Res. 2010;201:111-117.

11. Khan AZ, Heinen SJ, McPeek RM. Attentional cueing at the saccade goal, not at the target location, facilitates saccades. $J$ Neurosci. 2010;30:5481-5488.

12. Ditterich J, Eggert T, Straube A. Relation between the metrics of the presaccadic attention shift and of the saccade before 
and after saccadic adaptation. J Neurophysiol. 2000;84:18091813.

13. McLaughlin SC. Parametric adjustment in saccadic eye movements. Percept Psychophys. 1967;2:359-362.

14. Montagnini A, Chelazzi L. The urgency to look: prompt saccades to the benefit of perception. Vision Res. 2005;45: 3391-3401.

15. Glimcher PW. The neurobiology of visual-saccadic decision making. Ann Rev Neurosci. 2003;26:133-179.

16. Sheliga BM, Riggio L, Craighero L, Rizzolatti G. Spatial attention-determined modifications in saccade trajectories. Neuroreport. 1995;6:585-588.

17. Doyle M, Walker R. Curved saccade trajectories: voluntary and reflexive saccades curve away from irrelevant distractors. Exp Brain Res. 2001;139:333-344.

18. McSorley E, Haggard P, Walker R. Distractor modulation of saccade trajectories: spatial separation and symmetry effects. Exp Brain Res. 2004;155:320-333.

19. Gerardin P, Miquee A, Urquizar C, Pelisson D. Functional activation of the cerebral cortex related to sensorimotor adaptation of reactive and voluntary saccades. NeuroImage. 2012;61:1100-1112.

20. Panouilleres M, Habchi O, Gerardin P, et al. A role for the parietal cortex in sensorimotor adaptation of saccades. Cerebral Cortex. 2014;24:304-314.

21. Ditterich J, Eggert T, Straube A. The role of the attention focus in the visual information processing underlying saccadic adaptation. Vision Res. 2000;40:1125-1134.
22. Pelisson D, Alahyane N, Panouilleres M, Tilikete C. Sensorimotor adaptation of saccadic eye movements. Neurosci Biobehav Rev. 2010;34:1103-1120.

23. Bastian AJ. Understanding sensorimotor adaptation and learning for rehabilitation. Curr Opin Neurol. 2008;21:628633.

24. Panouilleres M, Gaveau V, Socasau C, Urquizar C, Pelisson D. Brain processing of visual information during fast eye movements maintains motor performance. PLoS One. 2013; 8:e54641.

25. Khan A, McFadden SA, Harwood M, Wallman J. Salient distractors can induce saccade adaptation. J Ophthalmol. 2014;2014:585792.

26. Schutz AC, Kerzel D, Souto D. Saccadic adaptation induced by a perceptual task. $J$ Vis. 2014;14:4.

27. McFadden SA, Khan A, Wallman J. Gain adaptation of exogenous shifts of visual attention. Vision Res. 2002;42: 2709-2726.

28. Smith DT, Schenk T, Rorden C. Saccade preparation is required for exogenous attention but not endogenous attention or IOR. J Exp Psychol Hum Percept Perform. 2012;38:1438-1447.

29. Bisley JW. The neural basis of visual attention. J Physiol. 2011; 589:49-57.

30. Culham JC, Cavanagh P, Kanwisher NG. Attention response functions: characterizing brain areas using fMRI activation during parametric variations of attentional load. Neuron. 2001;32:737-745. 